\title{
Société Suisse de Gastro-Entérologie
}

Assemblée annuelle les 29 et 30 octobre 1965 à Geneve

Schweizerische Gesellschaft für Gastroenterologie

Jahresversammlung in Genf, 29. und 30. Oktober 1965

Communications - Verzeichnis der Vorträge

Monod, M. E. (Avignon): Contribution à Tétude de la pathogénie des cholécystatonies 130

Sayegh, C; Demole, M. et Moïn, M. (Geneve): A propos des délais d'apparition radiologique des calculs vésiculaires 137

Rohner, A.; Bernhardt, J. P. et Perrier, C. (Geneve): Diaphragme duodenal congenital avec abouchement cholédoco-wirsungien ectopique; resec

tion, réimplantation papillaire, guérison 144

Chanal, G. (Geneve): Pseudo-Kyste du Pancreas 154

Affolter, H. (Basel): Die Rolle der Säureperfusion in der Diagnostik der Reflux-Osophagitis 157

Kahl, E. und Koch, E, (Frankfurt): Hiatushernien bei Kyphosen und Skoliosen 165

Arnold, J. et Hofstetter, J. R. (Lausanne): Correlations entre le sondage gastrique (selon Lambling) et la biopsie gastrique dans la gastrite 171

Hegglín, J.; Sumser, A. und Allgöwer, M. (Chur/St. Gallen): Beziehung der Antrum-Pylorusdysfunktion zum Ulcus duodeni und Ulcus ventriculi 180

La suite au prochain numéro - Schluss folgt im nächsten Heft

9 Gastroenterologia, Vol. 106, No. 3 (1966) 